\title{
Mongo Language
}

National Cancer Institute

\section{Source}

National Cancer Institute. Mongo Language. NCI Thesaurus. Code C154004.

A Niger-Congo Bantu language spoken by several of the Mongo peoples in the Democratic Republic of the Congo. 